\title{
A PURELY ANALYTIC CRITERION FOR A DECOMPOSABLE OPERATOR
}

\author{
by RIDGLEY LANGE
}

(Received 8 November, 1978)

In [3] E. Bishop introduced the notion of an operator with a "duality theory of type 3 " and gave a certain sufficient condition for an operator to have a duality theory of type 3. In this note we show that in fact Bishop's sufficient condition implies that a given operator is decomposable [4]. Moreover, this condition characterizes a decomposable operator.

Throughout this paper $X$ denotes a reflexive complex Banach space, and $T$ denotes a bounded linear operator on $X$. According to Bishop [3], Definition 5, T has a duality theory of type 3 if for each open cover $\left\{G_{1}, G_{2}, \ldots, G_{n}\right\}$ of the complex plane $\mathbf{C}$ there are invariant subspaces $M_{1}, \ldots, M_{n}$ which span $X$ such that $\sigma\left(T \mid M_{i}\right) \subset \bar{G}_{i}(i=1, \ldots, n)$. The above mentioned sufficient condition that $T$ have a duality theory of type 3 is that $T$ and its adjoint $T^{\prime}$ both have the following property $\beta$ ([3], p. 394).

$\beta$. If $f_{n}: D \rightarrow X$ is a sequence of analytic functions such that $(\lambda-T) f_{n}(\lambda) \rightarrow 0$ uniformly on $D$, then $\left\{f_{n}\right\}$ is uniformly bounded on compact subsets of $D$.

Decomposable operators are due to Foias [4] and may be defined as follows. First, the operator $T$ is said to have the single-valued extension property (SVEP) if zero is the only analytic function $f: D \rightarrow X$ for which $(\lambda-T) f(\lambda)=0$ for all $\lambda \in D$. In this case the spectral manifold $X_{T}(F)$ is defined for $F \subset \mathbf{C}$ as the set of $x \in X$ such that $x=(\lambda-T) f(\lambda)$ for $f$ analytic on $\mathbf{C} \backslash F$. Now $T$ is said to be decomposable if it has the SVEP and for each cover $\left\{G_{1}, \ldots, G_{n}\right\}$ of $\mathbf{C}$ the manifolds $X_{T}\left(\bar{G}_{i}\right)$ are closed $(i=1, \ldots, n)$ and $X=$ $X_{T}\left(\bar{G}_{1}\right)+\ldots+X_{T}\left(\bar{G}_{n}\right)$. Moreover, $\sigma\left(T \mid X_{T}\left(\bar{G}_{i}\right)\right) \subset \bar{G}_{i}$ for each $i$. Thus a decomposable operator has a duality theory of type 3, but the converse is false [1] (at least on nonreflexive spaces).

To prove the desired result we require two lemmas.

LEMma 1. If $T$ has property $\beta$, then $T$ has the SVEP.

Proof. Let $f: D \rightarrow X$ be analytic such that $(\lambda-T) f(\lambda)=0$ for $\lambda \in D$. Put $f_{n}(\lambda)=n f(\lambda)$, $n=1,2, \ldots$, and note that for $\lambda \in D$ fixed $\|n f(\lambda)\| \leq R$ for $R>0$ by $\beta$. Hence $f(\lambda)=0$ and $T$ has the SVEP.

By Lemma 1 the conclusion of the next lemma makes sense.

Lemma 2. Let $X^{\prime}$ be the dual space of $X$, and let $T^{\prime}$ denote the adjoint of $T$. If $T$ and $T^{\prime}$ both have property $\beta$, then $X_{T}(G)^{\perp}=X_{T^{\prime}}^{\prime}(\mathbf{C} \backslash G)$ for each open set $G$. In particular, $X_{T^{\prime}}^{\prime}(F)$ (dually, $X_{T}(F)$ ) is closed for $F$ closed.

Proof. First let $H, K$ be arbitrary disjoint sets in C. For $x \in X_{T}(H)$ and $u \in X_{T^{\prime}}^{\prime}(K)$ it follows by a straightforward application of Liouville's theorem that $\langle x, u\rangle$ (evaluation of $u$ at $x$ ) is 0 . Hence for any $G \subset \mathbf{C}$, we obtain $X_{T}(G)^{\perp} \supset X_{T^{\prime}}^{\prime}(\mathbf{C} \backslash G)$.

Glasgow Math. J. 21 (1980) 69-70. 
We next prove the reverse inclusion. Let $G$ be open, and let $H$ and $K$ be two open sets such that $\bar{H} \subset G$ and such that $\{G, K\}$ and $\{H, K\}$ separately cover $\mathbf{C}$. By [3], Theorems 3 and 4, p. 394, and Definition 3, p. 381, and the evident fact that $X_{T}(\bar{H}) \subset$ $X_{T}(G)$, we obtain the inclusions $X_{T}(G)^{\perp} \subset X_{T}(\bar{H})^{\perp} \subset X_{T^{\prime}}^{\prime}(\bar{K})$. Now let $\left\{K_{i}\right\}$ be a sequence of open sets such that $\left\{G, K_{j}\right\}$ covers $\mathbf{C}$ for $j=1,2, \ldots$, and $\mathbf{C} \backslash G=\cap \bar{K}_{j}$. By the last inclusion $X_{T}(G)^{\perp} \subset \cap X_{T^{\prime}}^{\prime}\left(\bar{K}_{j}\right)=X_{T^{\prime}}^{\prime}\left(\cap \bar{K}_{j}\right)=X_{T^{\prime}}^{\prime}(\mathbf{C} \backslash G)$, since $X_{T}(\quad)$ preserves intersections. Thus $X_{T}(G)^{\perp}=X_{T^{\prime}}^{\prime}(\mathbf{C} \backslash G)$.

THEOREM. Let $X$ be reflexive with dual $X^{\prime}$, and let $T$ be an operator on $X$ with adjoint $T^{\prime}$. Then $T$ is decomposable if and only if $T$ and $T^{\prime}$ both have property $\beta$.

Proof. Suppose $T$ and $T^{\prime}$ both have property $\beta$. By a recent result of Radjabalipour [7], it is enough to prove that $T$ is 2-decomposable, i.e. $X=X_{T}\left(\bar{G}_{1}\right)+X_{T}\left(\bar{G}_{2}\right)$ and $X_{T}\left(\bar{G}_{i}\right)$ are closed whenever $G_{1}, G_{2}$ cover $\mathbf{C}$. Let $\left\{G_{1}, G_{2}\right\}$ be such a cover, so that $H_{i}=\mathbf{C} \backslash \bar{G}_{i}$ $(i=1,2)$ have disjoint closures. By [2], Lemma 2.3, and Lemma $2, X_{T^{\prime}}^{\prime}\left(\bar{H}_{1}\right)+X_{T^{\prime}}^{\prime}\left(\bar{H}_{2}\right)$ is a direct sum, hence $X_{T^{\prime}}^{\prime}\left(H_{1}\right)^{-}+X_{T^{\prime}}^{\prime}\left(H_{2}\right)^{-}$is also direct. It is not hard to prove that $X_{T^{\prime}}^{\prime}\left(H_{1}\right)^{\perp}+X_{T^{\prime}}^{\prime}\left(H_{2}\right)^{\perp}=X$ (see [6, p. 1057]). By Lemma 2 (applied to $T^{\prime}$ ) $X=$ $X_{T}\left(\bar{G}_{1}\right)+X_{T}\left(\bar{G}_{2}\right)$ and the latter are closed. Hence $T$ is decomposable.

Conversely, let $T$ be decomposable. Then $T$ has property $\beta$ by [5], and $T^{\prime}$ is 2-decomposable by [6], Theorem 2; hence $T^{\prime}$ also has property $\beta$ by [5], final remark This completes the proof.

\section{REFERENCES}

1. E. Albrecht, An example of a weakly decomposable operator which is not decomposable Rev. Roumaine Math. Pures Appl. 20 (1975), 855-861.

2. C. Apostol, Roots of decomposable operator-valued analytic function, Rev. Roumain Math. Pures Appl. 13 (1968), 147-150.

3. E. Bishop, A duality theory for an arbitrary operator, Pacific J. Math. 9 (1959), 379-397.

4. C. Foias, Spectral maximal spaces and decomposable operators in Banach spaces, Arch Math. (Basel) 14 (1963), 341-349.

5. C. Foias, On the maximal spectral spaces of a decomposable operator, Rev. Roumain Math. Pures. Appl. 15 (1970), 1599-1606.

6. S. Frunza, A duality theorem for decomposable operators, Rev. Roumaine Math. Pure Appl. 16 (1971), 1055-1058.

7. M. Radjabalipour, Equivalence of decomposable and 2-decomposable operators, Pacific $J$ Math., 28 (1978), 243-247

Department of Mathematics

UNIVERSITY OF NEW ORLEANS

New Orleans, Louisiana 70122
Present address:

DePaRTMENT OF Mathematics

Youngstown State Universit

YOUNGSTOWN, OHIO 44555 